\title{
Einleitung der Herausgeber
}

\section{Die Kritische Schleiermacher-Gesamtausgabe}

Die Kritische Gesamtausgabe (KGA) der Schriften, des Nachlasses und des Briefwechsels Schleiermachers ist in die folgenden fünf Abteilungen gegliedert:

I. Schriften und Entwürfe

II. Vorlesungen

III. Predigten

IV. Übersetzungen

V. Briefwechsel und biographische Dokumente.

Die Gliederung richtet sich nach den literarischen Gattungen in Schleiermachers Werk, wobei den einzelnen Abteilungen jeweils auch der handschriftliche Nachlass zugewiesen wird. Der Aufbau der Abteilungen orientiert sich am chronologischen Prinzip.

\section{Die Abteilung II (Vorlesungen)}

Die II. Abteilung dokumentiert Schleiermachers Vorlesungstätigkeit nach seinen handschriftlichen Materialien und nach Vorlesungsnachschriften. Schleiermacher hat in seiner beinahe drei Jabrzehnte währenden Lehrtätigkeit in der Theologischen Fakultät, abgesehen vom Alten Testament, über nahezu alle theologischen Disziplinen Vorlesungen gehalten. Als Mitglied der Königlichen Akademie der Wissenschaften zu Berlin hatte er überdies das Recht, auch in der Philosophischen Fakultät Vorlesungen zu halten. Davon hat er extensiven Gebrauch gemacht. In jedem Semester hat Schleiermacher mindestens zwei Vorlesungen gehalten, oft sogar drei (eine neutestamentlich-exegetische, eine weitere theologische und eine philosophische). Ein Verzeichnis seiner Vorlesungen findet sich in dem von Andreas Arndt und Wolfgang Virmond bearbeiteten Band „Schleiermachers Briefwechsel (Verzeichnis) nebst einer Liste seiner Vorlesungen" (SchleiermacherArchiv Bd. 11, Berlin und New York 1992, S. 293-330). 
Angesichts der umfänglichen Materialien ist eine restriktive Berücksichtigung der Vorlesungsnachschriften unumgänglich. Für die Edition der Vorlesungen gelten folgende Richtlinien:

1. Jede von Schleiermacher in seinen Vorlesungen behandelte Disziplin wird in einem Band - eventuell mit Teilbänden vorrangig durch seine eigenen Manuskripte kritisch ediert.

2. Die Manuskripte Schleiermachers werden im ersten Teil in chronologischer Ordnung kritisch ediert.

3. Die Vorlesungsnachschriften werden, wenn ibre Qualität es erlaubt, dort in die Edition einbezogen und unter vereinfachten Editionsregeln in einem zweiten Teil ediert, wo eigene Manuskripte Schleiermachers entweder fehlen oder wo seine Manuskripte als nicht ausreichend zu beurteilen sind. Nachschriften eines mehrfach gehaltenen Kollegs aus verschiedenen Jahren werden nur dann eigens berücksichtigt, wenn es darum geht, eine bedeutsame Entwicklung zu dokumentieren. Auch die Nachschriften werden chronologisch angeordnet.

4. Die abgrenzende Gruppierung der Manuskripte Schleiermachers und der Nachschriften von fremder Hand in zwei Teilen des Bandes kann bei besonderen Sachlagen aufgegeben werden; die zu edierenden Texte werden dann fortlaufend chronologisch angeordnet.

Für die chronologische Anordnung der Vorlesungsdisziplinen ist dasjenige Semester maßgebend, in dem Schleiermacher die jeweilige Vorlesung zum ersten Mal gehalten hat. In den beiden Fällen, in denen er im selben Semester mit zwei bzw. drei Vorlesungen begonnen hat (Wintersemester 1804/05 und Sommersemester 1806), werden zuerst die allgemeiner und dann die spezieller ausgerichteten Vorlesungen geboten. Dementsprechend ergibt sich für die Abteilung „Vorlesungen" folgende Gliederung:

1. Vorlesungen über die Philosophische Sittenlehre (1804/05)

2. Vorlesungen über die Theologische Enzyklopädie (1804/05)

3. Vorlesungen über die Christliche Glaubenslehre (1804/05)

4. Vorlesungen zur Hermeneutik und Kritik (1805)

5. Vorlesungen über die Christliche Sittenlehre (1806)

6. Vorlesungen über die Kirchengeschichte (1806)

7. Vorlesungen über die Geschichte der griechischen Philosophie (1807) 
8. Vorlesungen über die Lehre vom Staat (1808/09)

9. Vorlesungen über die Geschichte der christlichen Philosophie (1810)

10. Vorlesungen über die Dialektik (1811)

11. Vorlesungen über die Praktische Theologie (1812)

12. Vorlesungen über die Pädagogik und amtliche Voten zum öffentlichen Unterricht (1813)

13. Vorlesungen über die Psychologie (1818)

14. Vorlesungen über die Ästhetik (1819)

15. Vorlesungen über das Leben Jesu (1819/20)

16. Vorlesungen über die Kirchliche Geographie und Statistik (1827)

17. Vorlesungen über die Einleitung in das Neue Testament (1829).

Die exegetischen Vorlesungen Schleiermachers werden aus pragmatischen Gründen an den Schluss der Abteilung gestellt, weil dazu sebr umfängliche Manuskripte Schleiermachers im Nachlass erhalten sind. Die Quantität und Qualität dieser Materialien stellen eine editorische Erschließung vor spezifische Probleme. Geplant ist, die Bandeinteilung an dem bei Schleiermacher erkennbaren Kurs über sechs Semester zu orientieren:

1. Vorlesungen über die Schriften des Lukas (Evangelium und Apostelgeschichte)

2. Vorlesungen über die Briefe des Apostels Paulus A

3. Vorlesungen über die Briefe des Apostels Paulus $B$

4. Vorlesungen über die Katholischen Briefe und den Brief an die Hebräer

5. Vorlesungen über das Evangelium des Johannes

6. Vorlesungen über das Evangelium des Matthäus.

\section{Editorische Grundsätze für die Abteilung II (Vorlesungen)}

Die folgenden Grundsätze schließen sich an die für die I. Abteilung in der Fassung von KGA I/1 und für die V. Abteilung in der Fassung von KGA V/1 niedergelegten an, tragen aber den Besonderbeiten der Vorlesungsedition Rechnung. 


\section{Historische Einführung und Editorischer Bericht}

Den Bänden der II. Abteilung wird jeweils eine Einleitung des Bandherausgebers vorangestellt, die eine Historische Einfübrung und einen Editorischen Bericht umfasst. Die Historische Einführung gibt Auskunft über die Entstehungs- und Überlieferungsgeschichte der jeweiligen Vorlesung. Gegebenenfalls wird über die Rezeption durch die Zeitgenossen berichtet. Der Editorische Bericht beschreibt die Materiallage und erläutert das editorische Verfahren.

\section{Textgestaltung und textkritischer Apparat}

Die Bände der II. Abteilung umfassen

(A) sämtliche Vorlesungsmanuskripte Schleiermachers

(B) dort, wo es zu deren Verständnis nötig ist oder wo andere Gründe es nabelegen, auch ausgewählte Vorlesungsnachschriften und ferner, falls keine solchen Primärquellen mehr vorhanden sind,

(C) auch Texte, die nur noch sekundär, etwa im Druck der „Sämmtlichen Werke“, vorliegen.

Für die Edition aller drei Sorten von Textzeugen gelten folgende Prinzipien:

a) Schreibweise und Zeichensetzung des zu edierenden Textzengen werden grundsätzlich beibehalten. Dies gilt auch für Schwankungen in der Schreibweise, bei denen es häufig eine Ermessensfrage darstellt, ob eine irrtümliche Schreibweise vorliegt. Hingegen werden Verschiedenheiten in der Verwendung von Zeichen (z. B. für Abkürzungen und Auslassungen), soweit sie willkürlich und sachlich obne Bedeutung sind, stillschweigend vereinheitlicht. Die von Schleiermacher für Randnotizen gebrauchten Verweiszeichen (Ziffern, Sterne, Kreuze etc.) werden einheitlich durch Ziffern wiedergegeben, sofern diese Randnotizen hier als Fußnoten wiedergegeben werden.

b) Offenkundige Schreibfebler oder Versehen werden im Text korrigiert. Im Apparat wird-ohne weitere Angabedie Schreibweise des Originals angeführt. 
c) Wo der Zustand des Textes eine Konjektur notwendig macht, wird diese im Text durchgeführt und im Apparat nachgewiesen; in Zweifelsfällen wird die Konjektur mit der Angabe „Kj" nur im Apparat vorgeschlagen. Wo bereits Konjekturen eines früheren Herausgebers vorliegen, werden diese unter Nennung des jeweiligen Urhebers und der Seitenzahl seiner Ausgabe oder Schrift im Apparat mitgeteilt. Wird eine solche Konjektur in den Text übernommen, so wird dies ebenfalls im Apparat nachgewiesen.

Über diese gemeinsamen Prinzipien hinaus wird für die drei unterschiedlichen Textsorten (Manuskripte Schleiermachers, Vorlesungsnachschriften und sekundäre Überlieferung) das im Folgenden beschriebene abgestufte Editionsverfahren angewandt.

\section{(A) Manuskripte Schleiermachers}

d) Es wird die letztgültige Textgestalt des Manuskripts wiedergegeben. Alle Belege für den Entstehungsprozess (wie Streichungen, Korrekturen, Umstellungen) werden im textkritischen Apparat - nach Möglichkeit gebündelt - mitgeteilt.

e) Zusätze zum ursprünglichen Text, die Schleiermacher eindeutig einverwiesen hat, werden in den laufenden Text eingefügt. Sie werden mit der Formel "mit Einfügungszeichen“ und mit Angabe des ursprünglichen Ortes im Manuskript im textkritischen Apparat nachgewiesen. Ist ein Zusatz von Schleiermacher nicht eingewiesen, aber seine eindeutige Einordnung in den Grundtext durch Sinn oder Position möglich, so wird im textkritischen Apparat nur der Ort angegeben. Zusätze, die sich nicht eindeutig in den Grundtext einfügen lassen, werden auf den jeweiligen Seiten - vom übrigen Text deutlich abgesetzt - unter Angabe des Ortes im Manuskript wiedergegeben.

f) Bei Abbreviaturen (Abkürzungen, Kontraktionen, Kürzeln), deren Sinn eindeutig ist, werden unter Weglassung eines evtl. vorhandenen Abkürzungszeichens (Punkt, Abkürzungsschleife usw.) die fehlenden Buchstaben im Text kursiv ergänzt. Chiffren für Wörter (z. B. $\Theta$ für Gott) werden ebenfalls im Text kursiv aufgelöst und im Abkürzungsverzeichnis zusammengestellt. Abbreviaturen und Chiffren, deren Auflösung unsicher ist, werden im Text belassen; für sie wird ggf. im textkritischen Apparat ein Vorschlag mit der Formel 
„Abk. wobl für ..." gemacht. Zur Zeit Schleiermachers geläufige Abkürzungen werden nicht aufgelöst. Soweit sie heute nicht mehr geläufig sind, werden sie im Abkürzungsverzeichnis mit ibren Auflösungen zusammengestellt. Die durch Überstreichung bezeichnete Verdoppelung von $m$ und $n$ wird stillschweigend ausgeschrieben. In allen Fällen, wo (z. B. bei nicht ausgeformten Buchstaben, auch bei der verkürzten Endsilbe -en) aufgrund der Flüchtigkeit der Schrift nicht eindeutig ein Schreibversehen oder eine gewollte Abkürzung zu erkennen ist, wird das betreffende Wort obne weitere Kennzeichnung in der üblichen Schreibweise vollständig wiedergegeben.

g) Fehlende Wörter und Zeichen, die für das Textverständnis unentbehrlich sind, werden in eindeutigen Fällen kursiv in eckigen Klammern ergänzt. In Zweifelsfällen wird im Apparat mit der Formel ,zu ergänzen wohl“ ein Vorschlag gemacht. Im Text gelassene Lücken werden im textkritischen Apparat durch den Hinweis (lacuna) gekennzeichnet. Sofern das Zeilenende bzw. das Ende eines Absatzes eindeutig den Punkt am Satzende vertritt, wird dieser stillschweigend ergänzt. Ferner werden fehlende Umlautzeichen in eindeutigen Fällen stillschweigend ergänzt; fehlende diakritische Zeichen (wie Akzente, Spiritus-Zeichen) in fremdsprachigen Texten werden hingegen nicht ergänzt.

b) Sind im Manuskript Umstellungen von benachbarten Wörtern oder Satzteilen vorgenommen worden, so wird im Apparat mit der Formel „umgestellt aus" die Vorstufe angegeben. Bei Umstellungen von Sätzen oder Satzteilen über einen größeren Zwischenraum wird der ursprüngliche Ort unter Verwendung der Formel „mit Umstellungszeichen“ angegeben.

i) Streichungen. Sind im Manuskript Wörter, Buchstaben oder Zeichen gestrichen worden, so wird das Gestrichene im Apparat in Winkelklammern unter Angabe des Ortes im Manuskript mitgeteilt. Wurden Streichungen vorgenommen, aber nicht vollständig durchgeführt, so werden die versehentlich nicht gestrichenen Partien in doppelte Winkelklammern eingeschlossen.

j) Korrekturen Schleiermachers an Wörtern, Wortteilen oder Zeichen werden durch die Formel „korr. aus" angezeigt (Beispiel: klein] korr. aus mein).

k) Unsichere Lesarten werden in unvollständige eckige Klammern (Beispiel: [noch]) eingeschlossen. Gegebenenfalls 
wird eine mögliche andere Lesart mit der Formel "oder" (Beispiel: [auch oder [noch]) vorgeschlagen. Bei unsicheren Lesarten, zu denen frühere Texteditionen eine abweichende, ebenfalls erwägenswerte Lesart bieten, wird diese unter Nennung des jeweiligen Herausgebers und der Seitenzahl seiner Ausgabe oder Schrift mitgeteilt. Nicht entzifferte Wörter werden durch ein in unvollständige eckige Klammern gesetztes Spatium gekennzeichnet; bei zwei oder mehr unleserlichen Wörtern wird dieses Zeichen doppelt gesetzt und eine genauere Beschreibung im textkritischen Apparat gegeben

l) Liegen bei einer Handschriftenstelle mehrere deutlich unterscheidbare Entstehungsstufen vor, so können diese, wo es die Klarbeit erfordert, im textkritischen Apparat nacheinander jeweils für sich nachgewiesen werden. Keine eigene Mitteilung erfolgt, wenn beim Übergang aus der früheren in die spätere Stufe ein Wort gestrichen oder korrigiert worden ist; dieses ergibt sich aus dem Vergleich der Stufen.

m) Überlieferungslücken. Ist ein Manuskript nur bruchstückhaft überliefert, so wird der Überlieferungsverlust innerhalb eines Absatzes durch ein in kursive eckige Klammern eingeschlossenes Spatium gekennzeichnet. Ein umfangreicherer Überlieferungsverlust wird durch ein in kursive eckige Klammern gesetztes Spatium gekennzeichnet, das auf einer gesonderten Zeile wie ein Absatz eingerückt wird. Eine Beschreibung erfolgt im textkritischen Apparat.

\section{(B) Vorlesungsnachschriften}

Die Edition der Vorlesungsnachschriften erfolgt nach einem vereinfachten Verfahren. Diese Vereinfachungen betreffen die im Vorstehenden unter den Buchstaben d), e), b), i), j) und l) genannten Editionsregeln. Die unter den Buchstaben f), g), $k$ ) und $m$ ) genannten Grundsätze gelten unverändert.

n) Bei der Edition von Vorlesungsnachschriften wird in der Regel lediglich die letztgültige Textgestalt wiedergegeben, jedoch obne Nachweis des Manuskriptbefundes - d.i. von Streichungen, Zusätzen, Verbesserungen, Umstellungen und Entstehungsstufen - im Apparat. Abweichend hiervon werden längere Randbemerkungen zu Vorlesungsnachschriften, die den Charakter von eigenständigen Textpartien haben, 
als Fußnoten mitgeteilt, da es sich bei ibnen um spätere Ergänzungen des Nachschreibers handeln kann.

o) Existieren zu einer Vorlesung mehrere Nachschriften, so wird die beste als Leittext ediert. Die als Leittext gewählte Nachschrift wird in der Regel vollständig geboten. Wo Vorlesungsnachschriften über Schleiermachers Manuskripte hinaus keine wesentlichen Aufschlüsse enthalten, ist es auch möglich, sie nur ausschnittweise abzudrucken. Bietet die als Leittext gewählte Nachschrift an einer Stelle einen offenkundig fehlerhaften Text, so wird nach Möglichkeit der richtige Text aus einer anderen Nachschrift übernommen, die Abweichung aber im Apparat dokumentiert. Ist die als Leittext gewählte Nachschrift unvollständig, wird sie aus einer vollständigeren ergänzt, mit entsprechendem Nachweis im Apparat. Weist auch diese offenkundige Fehler auf, wird, sofern weitere Vorlesungsnachschriften vorhanden sind, verfahren wie im vorigen Satz beschrieben.

\section{(C) Sekundäre Überlieferung}

p) Sofern Überlieferungsverluste gegenüber früheren Editionen eingetreten sind, können die entsprechenden Texte als sekundäre Überlieferung in ibrer ursprünglichen Gestalt unverändert unter Hinzufügung eines Sachapparats dargeboten werden.

\section{Sachapparat}

Der Sachapparat gibt die für das Textverständnis notwendigen Erläuterungen.

a) Zitate und Verweise werden im Apparat nachgewiesen. Dabei wird, soweit möglich und sinnvoll, sowohl die von Schleiermacher benutzte Ausgabe als auch eine heute maßgebliche Ausgabe angeführt. Das gilt auch für Verweisungen Schleiermachers auf eigene Werke. Bei Zitaten werden sinnverändernde Abweichungen von den Quellen vermerkt.

b) $\mathrm{Zu}$ Anspielungen Schleiermachers werden Nachweise oder Erläuterungen nur dann gegeben, wenn die Anspielung als solche deutlich, der fragliche Sachverhalt eng umgrenzt und eine Erläuterung zum Verständnis des Textes nötig ist. 


\section{Verzeichnisse und Register}

a) Jeder Band erhält ein Abkürzungsverzeichnis, das sämtliche Zeichen und Abkürzungen auflöst, die von den Autoren oder vom Bandherausgeber benutzt worden sind, soweit die Auflösung nicht in den Apparaten oder im Literaturverzeichnis erfolgt.

b) Jeder Band erhält ein Literaturverzeichnis, in dem die Schriften aufgefübrt werden, die in den Texten sowie in den Apparaten und in der Einleitung des Bandherausgebers genannt sind. Bei denjenigen Werken, die im Katalog der Bibliothek Schleiermachers (s. Günter Meckenstock: Schleiermachers Bibliothek nach den Angaben des Rauchschen Auktionskatalogs und der Hauptbücher des Verlages G. Reimer, in: KGA I/15, 2005, S. 637-912) verzeichnet sind, wird nach dem Titel in eckigen Klammern das Kürzel SB mit der jeweiligen Katalognummer hinzugefügt.

c) Jeder Band erhält ein Namenregister, das alle im Band genannten historischen Personen erfasst.

d) Ein Register der Bibelstellen erhalten diejenigen Bände, bei denen es sinnvoll ist.

\section{Druckgestaltung}

a) Satzspiegel. Es werden untereinander angeordnet: Text des Originals ggf. mit Fußnoten, textkritischer Apparat, Sachapparat.

b) Schriftarten. Der Text des Originals wird einheitlich in recte stehender Antiqua wiedergegeben. Hochgestellte Endungen (z. B. bei Ordnungszahlen) werden nivelliert, graphische Varianten von Zeichen (wie doppelte Bindestriche, verschiedene Formen von Abkürzungszeichen oder Klammern) werden stillschweigend vereinheitlicht. Ergänzungen nicht ausgeschriebener Wörter im Text sowie Herausgeberrede werden kursiv gesetzt.

c) Hervorbebungen in Schleiermachers Manuskripten (vorwiegend durch Unterstreichung) werden einheitlich durch Sperrung kenntlich gemacht. Hervorbebungen in den Vorlesungsnachschriften bleiben unberücksichtigt, soweit sie der Lesbarkeit nicht förderlich sind.

d) Die Seitenzäblung des Originals wird auf dem Außenrand angegeben. Stammt die Zählung nicht vom Autor, so 
wird sie kursiv gesetzt. Der Seitenwechsel des zugrundeliegenden Textzeugen wird im Text durch einen senkrechten Strich (I) wiedergegeben. Wo die Angabe des Zeilenbruchs sinnvoll erscheint, erfolgt sie durch einen Schrägstrich (/) im Text.

e) Sofern ein Text bereits in den Sämmtlichen Werken erschienen ist, wird die Paginierung kursiv am Außenrand mitgeteilt, jedoch obne Seitentrennungsstrich.

f) Beziehung der Apparate auf den Text. Sie erfolgt beim textkritischen Apparat durch Zeilenangabe mit Lemma. Kommt in einer Zeile das gleiche Bezugswort mehrfach vor, wird ein zusätzliches Bezugswort angeführt. Die Bezugswörter werden durch das Lemmazeichen von der folgenden Mitteilung abgegrenzt. Der Sachapparat wird durch Zeilenangabe auf die jeweilige Bezugsstelle bezogen.

g) Sofern in einem Band sowohl Manuskripte Schleiermachers als auch eine Nachschrift aus demselben Kolleg veröffentlicht werden, wird der Zusammenhang zwischen ihnen möglichst durch ein Verweisungssystem hergestellt, etwa durch die Angabe der Daten oder durch die Bezeichnung der Vorlesungsstunden am Seitenrand. Sofern solche Angaben in den edierten Quellen enthalten sind, werden sie recte wiedergegeben; sofern sie aus anderen Quellen ergänzt sind, werden sie kursiv gesetzt.

Im Namen der Herausgeber Lutz Käppel 